\title{
Parameter-dependent transmission dynamics and optimal control of foot and mouth disease in a contaminated environment
}

\author{
Francis Mugabi ${ }^{1 *}$ (D), Joseph Mugisha ${ }^{1}$, Betty Nannyonga ${ }^{1}$, Henry Kasumba $^{1}$ and Margaret Tusiime ${ }^{2}$
}

\author{
*Correspondence: \\ mugabikanywa@yahoo.com \\ ${ }^{1}$ College of Natural Sciences, \\ Makerere University, Wandegeya, P. \\ O. Box 7062 Kampala, Uganda \\ Full list of author information is \\ available at the end of the article
}

\begin{abstract}
The problem of foot and mouth disease (FMD) is of serious concern to the livestock sector in most nations, especially in developing countries. This paper presents the formulation and analysis of a deterministic model for the transmission dynamics of FMD through a contaminated environment. It is shown that the key parameters that drive the transmission of FMD in a contaminated environment are the shedding, transmission, and decay rates of the virus. Using numerical results, it is depicted that the host-to-host route is more severe than the environmental-to-host route. The model is then transformed into an optimal control problem. Using the Pontryagin's Maximum Principle, the optimality system is determined. Utilizing a gradient type algorithm with projection, the optimality system is solved for three control strategies: optimal use of vaccination, environmental decontamination, and a combination of vaccination and environmental decontamination. Results show that a combination of vaccination and environmental decontamination is the most optimal strategy. These results indicate that if vaccination and environmental decontamination are used optimally during an outbreak, then FMD transmission can be controlled. Future studies focusing on the control measures for the transmission of FMD in a contaminated environment should aim at reducing the transmission and the shedding rates, while increasing the decay rate.
\end{abstract}

Keywords: Parameter dependent, FMD, Contaminated environment, Optimal control

\section{Introduction}

Foot and mouth disease (FMD) is a highly contagious viral disease that affects all clovenhoofed domestic and wild animals [3]. FMD is caused by a virus of genus Aphthovirus of the family Picornaviridae [8]. Outbreaks of FMD are characterized by fever, drop in milk production, excessive salivation, and development of vesicles on the tongue, gums, teats, and feet [1]. The mortality rate of animals due to FMD is usually low but may be high depending on the age of the animals. The control measures against FMD include vaccination, quarantine, bio-security measures, and killing the infected animals [7].

FMD can be transmitted either directly through contact with an infected host [4] or indirectly through contact with a contaminated environment [9]. Direct transmission of FMD occurs when infected and susceptible animals get in close contact with each other.

(c) The Author(s). 2019 Open Access This article is distributed under the terms of the Creative Commons Attribution 4.0 International License (http://creativecommons.org/licenses/by/4.0/), which permits unrestricted use, distribution, and reproduction in any medium, provided you give appropriate credit to the original author(s) and the source, provide a link to the Creative Commons license, and indicate if changes were made. 
Indirect transmission of FMD through a contaminated environment occurs when the susceptible host comes into contact with the infected secretions and excretions. Once infected, the animal releases large amounts of the virus in secretions and excretions, before [30] and after [15] the appearance of clinical signs. Upon contact of a susceptible animal with infectious secretions and excretions, the infectious cycle continues. FMDV can remain in secretions and excretions for long periods of time and this facilitates the transmission of FMD through a contaminated environment. Traditionally, direct route of disease transmission has been considered as the main cause of infection spread. However, the role of indirect transmission through a contaminated environment is becoming more evident [6].

Numerous models have been developed to study the role of the environment in the transmission dynamics of different pathogens [10, 24, 28, 31] and both deterministic [14] and stochastic $[17,21]$ models have been used to study the transmission dynamics and control of FMD, but none of such studies shows a significant development in assessing the parameters that determine the transmission dynamics of FMD in a contaminated environment. In addition, it is known that vaccination [7] and environmental decontamination [18] are important tools in controlling the environmental transmission of FMD. However, no sufficient knowledge on the optimal way of applying these control tools exists. Thus, the aim of this paper is to formulate a mathematical model to determine the key parameters and optimal control measures for the transmission of FMD through a contaminated environment.

The rest of this paper is organized as follows. In the "Model formulation and analysis" section, formulation and analysis of the deterministic model for the transmission dynamics of FMD in a contaminated environment are done. In the "Analysis of optimal control measures for the environmental transmission of FMD" section, the optimal control strategies for the disease are explored. The paper ends with a discussion of the results in the "Discussion" section.

\section{Model formulation and analysis}

Considering the fact that the animals exposed to FMD can release large amounts of the virus into the environment [30], we extend a Susceptible-Infectious-RecoveredSusceptible-Pathogens (SIRSP) model presented in [6] to an SEIRSP model that includes Asymptomatic individuals $E$ capable of shedding FMDV into the environment. We ignore the growth term considered in [6] because viruses do not multiply outside their hosts.

Let the total cattle population at any time $t$ be denoted by $N(t)$. In this model, the cattle population is sub-divided into the Susceptible $S(t)$, Asymptomatic $E(t)$, Symptomatic $I(t)$, and Recovered $R(t)$ cattle. The FMDV pathogens in the environment are denoted by $P(t)$.

In the animal population, susceptible cattle $S(t)$ are recruited by birth at a constant rate $\Lambda$. Direct transmission is due to contact with susceptible $S(t)$ and infected symptomatic cattle $I(t)$ at a rate $\beta_{I}$. It is assumed that the process of direct transmission follows the law mass of action and therefore denoted by $\beta_{I} S I$. Indirect transmission is between a contaminated environment $P(t)$ and the susceptible individuals $\mathrm{S}(\mathrm{t})$. If $\beta_{p}$ is the rate of infection due to a contaminated environment, then $\beta_{p} P S$ is the force of infection from a contaminated environment to the susceptible subjects which is also modeled by the law of mass action. 
After infection, the cow is now exposed to FMD and transits to the asymptomatic class $E(t)$. At the end of the incubation period usually $\frac{1}{\sigma}$ days, the cow is now symptomatic and moves to class $I(t)$, where it can die due to FMD at a rate $v$ or may recover at a rate $\gamma$ to join the recovered class $R(t)$. While in $R(t)$, the cow gains temporary immunity for at least 6 months after which it moves back to $S(t)$ at a rate $\omega$ and the cycle continues. All the animal sub-groups are subjected to the per capita natural mortality rate $\mu$. Infected cattle shed FMDV into the environment at a rate of $\lambda_{p}$. FMDV pathogens in the environment decay due to the natural causes at a rate of $\delta_{p}$. The parameters with their values are given in the Table 1.

Combining all the above definitions and assumptions together, the system of the ordinary differential equations describing the dynamics of FMD in a contaminated environment is obtained and it is given by

$$
\begin{aligned}
& \frac{d S}{d t}=\Lambda+\omega R-\left(\beta_{p} P+\beta_{I} I\right) S-\mu S, \\
& \frac{d E}{d t}=\left(\beta_{p} P+\beta_{I} I\right) S-(\sigma+\mu) E, \\
& \frac{d I}{d t}=\sigma E-(\nu+\mu+\gamma) I, \\
& \frac{d R}{d t}=\gamma I-(\mu+\omega) R, \\
& \frac{d P}{d t}=\lambda_{p}(I+E)-\delta_{p} P .
\end{aligned}
$$

Summing up the equations for $S, E, I$, and $R$ we obtain

$$
\frac{d N}{d t}=\Lambda-\mu N-v I
$$

To determine whether the model is biologically meaningful, it is tested for positivity and boundedness.

Theorem 1 The solutions $S(t), E(t), I(t), R(t)$ and $P(t)$ of the model (1)-(5) with positive initial data, remain positive for all time $t>0$.

Proof Suppose that $t_{1}=\sup \{t>0: S(t)>0, E(t)>0, I(t)>0, R(t)>0, P(t)>0\}>$ 0 . It follows from (1) that

$$
\frac{d S}{d t}=\Lambda+\omega R-\left(\beta_{p} P+\beta_{I} I\right) S-\mu S \geq \Lambda-\left(\beta_{p} P+\beta_{I} I\right) S-\mu S
$$

Table 1 Model parameters and their values

\begin{tabular}{llll}
\hline Parameter & Description & Value & Reference \\
\hline$\Lambda$ & Recruitment rate & 0.2 & {$[6]$} \\
$\omega$ & Rate of immunity loss & 0.0056 & {$[13]$} \\
$\mu$ & Natural mortality rate of cattle & 0.0002 & {$[2]$} \\
$\beta_{p}$ & Environment-to-host transmission rate & 0.01 & Assumed \\
$\beta_{l}$ & Host-to-host transmission rate & 0.0001 & Assumed \\
$\sigma$ & Development rate of clinical signs & 0.25 & {$[17]$} \\
$v$ & Disease-induced mortality rate of cattle & 0.0064 & {$[11]$} \\
$\gamma$ & Recovery rate of infected cattle & 0.143 & {$[22]$} \\
$\lambda_{p}$ & Shedding rate of FMDV & 0.002 & Assumed \\
$\delta_{p}$ & Decay rate of FMDV & 0.0476 & Assumed \\
\hline
\end{tabular}


which can be written as

$$
\frac{d}{d t}\left(S(t) \exp \left[\mu t+\int_{0}^{t}\left(\beta_{p} P+\beta_{I} I\right)(u) d u\right]\right) \geq \Lambda \exp \left[\mu t+\int_{0}^{t}\left(\beta_{p} P+\beta_{I} I\right)(u) d u\right] .
$$

Hence,

$$
\begin{aligned}
& S\left(t_{1}\right) \exp \left[\mu t_{1}+\int_{0}^{t_{1}}\left(\beta_{p} P+\beta_{I} I\right)(u) d u\right] \\
& -S(0) \geq \int_{0}^{t_{1}}\left(\Lambda \exp \left[\mu y+\int_{0}^{y}\left(\beta_{p} P+\beta_{I} I\right)(u) d u\right]\right) d y,
\end{aligned}
$$

so that

$$
\begin{aligned}
S\left(t_{1}\right) \geq & S(0) \exp \left[-\mu t_{1}-\int_{0}^{t_{1}}\left(\beta_{p} P+\beta_{I} I\right)(u) d u\right] \\
& +\exp \left[-\mu t_{1}-\int_{0}^{t_{1}}\left(\beta_{p} P+\beta_{I} I\right)(u) d u\right] \\
& \int_{0}^{t_{1}}\left(\Lambda \exp \left[\mu y+\int_{0}^{y}\left(\beta_{p} P+\beta_{I} I\right)(u) d u\right]\right) d y>0 .
\end{aligned}
$$

Similarly, it can be shown that $E(t) \geq 0, I(t) \geq 0, R(t) \geq 0$ and $P(t) \geq 0$ for all time $t>0$.

Therefore, all solutions of the model (1)-(5) remain positive for all non-negative initial conditions.

Theorem 2 The solutions to the model (1)-(5) with non-negative initial data are bounded.

Proof From (6), we have

$$
\frac{d N}{d t} \leq \Lambda-\mu N
$$

This gives a solution of

$$
N(t) \leq \frac{\Lambda}{\mu}+\left\{N_{0}-\frac{\Lambda}{\mu}\right\} e^{-\mu t}
$$

Hence,

$$
\Omega_{N}=\left\{N(t) \in \mathbb{R}_{+}: N(t) \leq \frac{\Lambda}{\mu}\right\} .
$$

Therefore, all solutions to the model (1) -(5) with non-negative initial data are bounded. From (13), we obtain the feasible region

$$
\Omega=\left\{N(t) \in \mathbb{R}_{+}: N(t) \leq \frac{\Lambda}{\mu}\right\} .
$$

In this region, the model can be considered as being epidemiologically feasible, positively invariant and mathematically well-posed [16]. Therefore, every solution of the system with initial conditions in $\Omega$ always remains in $\Omega$ for all $t>0$. 


\section{Existence and local stability of disease-free equilibrium point $\left(D_{0}\right)$}

The model (1)-(5) has a disease-free equilibrium point $D_{0}$ given by

$$
D_{0}=\left(S^{*}, E^{*}, I^{*}, R^{*}, P^{*}\right)=\left(\frac{\Lambda}{\mu}, 0,0,0,0\right) .
$$

Definition 1 The point $\left(D_{0}\right)$ is said to be locally stable if all solutions of the model that start out any where in $\Omega$ stay near $D_{0}$ over a definite time.

The local stability of $D_{0}$ is explored using the next-generation operator method [29]. Consider the infectious compartments $E(t), I(t)$, and $P(t)$ of the model (1)-(5). Let $\mathcal{F}$ be the rate of appearance of new infections into the infectious compartments, $\mathcal{V}^{+}$be the rate of transfer of individuals into the infectious compartments, and $\mathcal{V}^{-}$be the rate of transfer of individuals out of the infectious compartments. From (1)-(5), it can be observed that the rate of appearance of new infections into the infectious compartments is given by

$$
\mathcal{F}=\left(\begin{array}{c}
\left(\beta_{P} P+\beta_{I} I\right) S \\
0 \\
\lambda_{p}(I+E)
\end{array}\right)
$$

the rate of transfer of individuals into the infectious compartments by

$$
\mathcal{V}^{+}=\left(\begin{array}{c}
0 \\
\sigma E \\
0
\end{array}\right)
$$

and the rate of transfer of individuals out of the infectious compartments by

$$
\mathcal{V}^{-}=\left(\begin{array}{c}
(\sigma+\mu) E \\
(\nu+\mu+\gamma) I \\
\delta_{p} P
\end{array}\right)
$$

Then, it follows that

$$
\mathcal{V}=\mathcal{V}^{-}-\mathcal{V}^{+}=\left(\begin{array}{c}
(\sigma+\mu) E \\
-\sigma E+(\nu+\mu+\gamma) I \\
\delta_{p} P
\end{array}\right)
$$

The Jacobian, $F$ of $\mathcal{F}$ at the disease-free equilibrium point is given by

$$
F=\left(\begin{array}{ccc}
0 & \beta_{I} \frac{\Lambda}{\mu} & \beta_{p} \frac{\Lambda}{\mu} \\
0 & 0 & 0 \\
\lambda_{p} & \lambda_{p} & 0
\end{array}\right)
$$

and the Jacobian, $V$ of $\mathcal{V}$ at the disease-free equilibrium point by

$$
V=\left(\begin{array}{ccc}
\sigma+\mu & 0 & 0 \\
-\sigma & \mu+\nu+\gamma & 0 \\
0 & 0 & \delta_{p}
\end{array}\right)
$$


It follows that the associated basic reproduction number [16], denoted by $\mathcal{R}_{0}$, is given by

$$
\begin{aligned}
\mathcal{R}_{0}=\rho\left(F V^{-1}\right)= & \frac{\beta_{I} \sigma \Lambda}{2 \mu(\sigma+\mu)(\mu+v+\gamma)}+\left(\left[\frac{\beta_{I} \sigma \Lambda}{2 \mu(\sigma+\mu)(\mu+v+\gamma)}\right]^{2}\right. \\
& \left.+\frac{\Lambda(\sigma+\mu+v+\gamma)}{\mu(\sigma+\mu)(\mu+v+\gamma)}\left[\frac{\lambda_{p} \beta_{p}}{\delta_{p}}\right]\right)^{\frac{1}{2}},
\end{aligned}
$$

where $\rho$ represents the spectral radius (the dominant eigenvalue in magnitude) of $F V^{-1}$. Using Theorem 2 of [29], the following result is established.

Lemma 1 The disease-free equilibrium point, $D_{0}$ is locally asymptotically stable in $\Omega$ whenever $\mathcal{R}_{0}<1$ and unstable if $\mathcal{R}_{0}>1$.

The threshold quantity $\mathcal{R}_{0}$ is the reproduction number for FMD model (1)-(5). It measures the average number of new cases generated by a single FMD infected cow in a population throughout its life time. In epidemics, $\mathcal{R}_{0}$ is useful in describing the magnitude of transmission. If $\mathcal{R}_{0}>1$, then the outbreak generates an epidemic, whereas if $\mathcal{R}_{0}<1$, then the infection will disappear from the population [6]. Equation 22 can be written as

$$
\mathcal{R}_{0}=\frac{1}{2}\left[\mathcal{R}_{d}+\sqrt{\mathcal{R}_{d}^{2}+4 \mathcal{R}_{p}}\right]
$$

where $\mathcal{R}_{d}=\frac{\beta_{I} \sigma \Lambda}{\mu(\sigma+\mu)(\mu+v+\gamma)}$, and $\mathcal{R}_{p}=\frac{\Lambda(\sigma+\mu+v+\gamma)}{\mu(\sigma+\mu)(\mu+v+\gamma)}\left[\frac{\lambda_{p} \beta_{p}}{\delta_{p}}\right]$. The quantities $\mathcal{R}_{d}$ and $\sqrt{\mathcal{R}_{p}}$ correspond, respectively, to the average number of secondary infections through host-to-host and environment-to-host transmission caused by one infectious cow in its infectious period. From (22), it can be seen that there is a positive relationship between $\mathcal{R}_{0}$ and the parameters $\beta_{I}, \beta_{p}$, and $\lambda_{p}$ (It can be shown that $\frac{\partial \mathcal{R}_{0}}{\partial \beta_{I}}>0, \frac{\partial \mathcal{R}_{0}}{\partial \beta_{p}}>0$ and $\frac{\partial \mathcal{R}_{0}}{\partial \lambda_{p}}>0$ ). This implies that these parameters have the potential of aggravating the infection when increased. In Fig. 1a-c, the effect of these parameters on the development of new infections of FMD $(E)$ is depicted. From this figure it is easy to visualize that increase in the transmission $\left(\beta_{I}\right.$ and $\left.\beta_{p}\right)$ and shedding $\left(\lambda_{p}\right)$ rates causes an increase in the development of new infections. From (22), it can be observed that there is a negative relationship between the decay rate parameter $\delta_{p}$ and $\mathcal{R}_{0}$ (It can be verified that $\frac{\partial \mathcal{R}_{0}}{\partial \delta_{p}}<0$ ). This means that $\delta_{p}$ has the ability of minimizing the infection when increased. From Fig. 1d, it is observed that increase in the decay rate $\delta_{p}$ leads to the decrease in the asymptomatic cattle $E$. From Fig. 1e, it can be noted that the host-to-host route of FMD leads to more infections as compared to the environment-to-host route.

\section{Global stability of the disease-free equilibrium point $\left(D_{0}\right)$}

Definition 2 The point $\left(D_{0}\right)$ is said to be globally stable if all solutions of the model that start out any where in $\Omega$ stay near $D_{0}$ over an indefinite time.

Motivated by Shuai and van den Driessche [25], the global stability of $D_{0}$ is analysed by using a Lyapunov function. A function $\mathcal{L}$ is called a Lyapunov function for the model $(1)-(5)$ if it is continuous and non-increasing along every solution of the system. To construct a Lyapunov function, a matrix theoretic method based on the Perron eigenvector is used [25]. For other methods of Lyapunov construction see [26]. Let $x=(E, I, P)^{T}$ and 
(a)

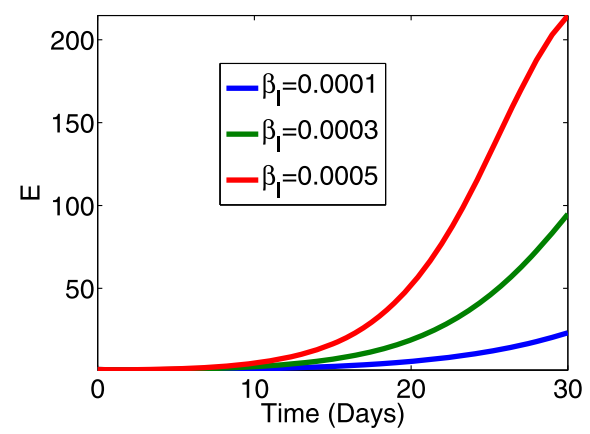

(b)

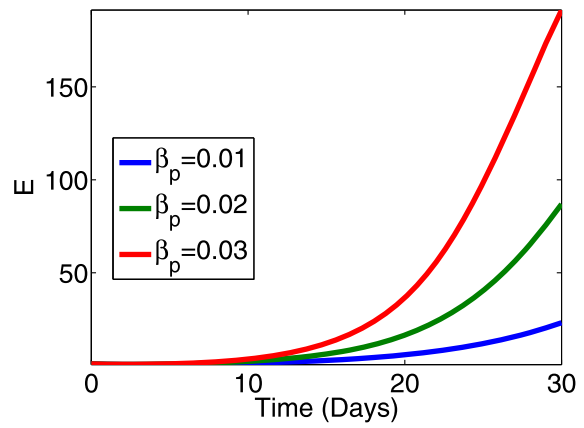

(c)

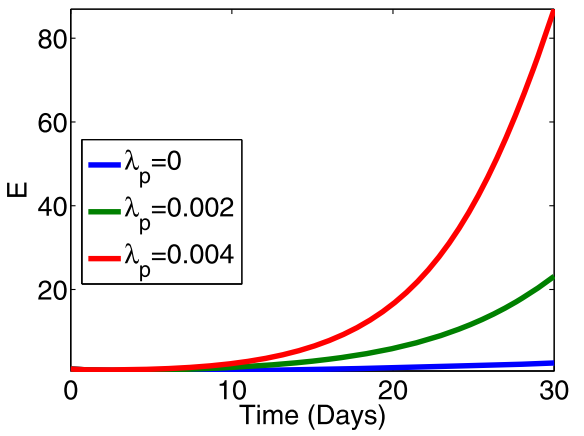

(d)

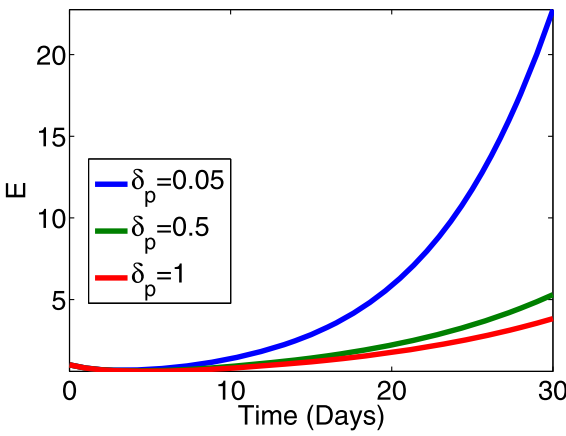

(e)

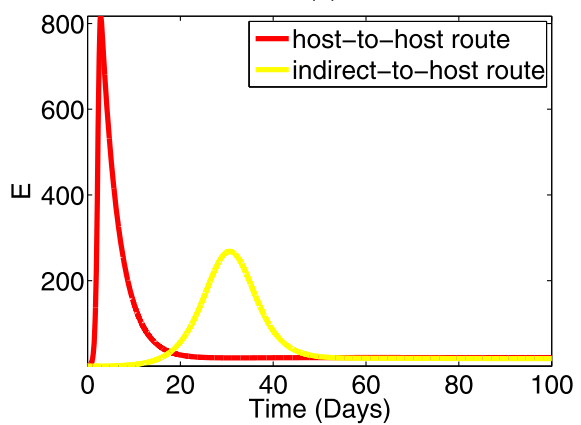

Fig. 1 a-e Variation of $E$ with time for different values of the parameters $\beta_{l,} \beta_{p}, \delta_{p}$ and $\lambda_{p}$. In $\mathbf{e} \beta_{l}=0.05$ and $\beta_{p}=0.05$. Other parameter values are in Table (1)

$y=(S, R)^{T}$ represent the populations in the infectious and non-infectious compartments, respectively. Generally, the model (1)-(5) can be represented as

$$
\begin{aligned}
& \frac{d x}{d t}=\mathcal{F}(x, y)-\mathcal{V}(x, y), \\
& \frac{d y}{d t}=g(x, y),
\end{aligned}
$$

with $g=\left(g_{1}, g_{2}\right)^{T}$, where $\mathcal{F}, \mathcal{V}^{+}$and $\mathcal{V}^{-}$are defined, respectively, as in (16), (17), and (18). Set

$$
f(x, y)=(F-V) x-\mathcal{F}(x, y)+\mathcal{V}(x, y),
$$


where $F$ and $V$ are defined, respectively, as in (20) and (21). Then, Eq. 24 for the diseased compartments can be written as

$$
\frac{d x}{d t}=(F-V) x-f(x, y)
$$

Let $\xi^{T} \geq 0$ be the left eigenvector of the non-negative matrix $V^{-1} F$ corresponding to the eigenvalue $\rho\left(V^{-1} F\right)=\rho\left(F V^{-1}\right)=\mathcal{R}_{0}$ [25], then, the Lyapunov function, $\mathcal{L}$ for the model (1)-(5) is given by the following theorem.

Theorem 3 Let $F, V$ and $f(x, y)$ be defined, respectively, as in (20), (21), and (26). If $f(x, y) \geq 0$ in $\Omega, F \geq 0, V^{-1} \geq 0$, and $\mathcal{R}_{0} \leq 1$, then $\mathcal{L}=\xi^{T} V^{-1} x$ is a Lyapunov function for the model (1)-(5) in $\Omega$.

Proof Differentiating $\mathcal{L}$ along the solutions of the model (1)-(5) gives

$$
\begin{aligned}
\mathcal{L}^{\prime} & =\xi^{T} V^{-1} x^{\prime}=\xi^{T} V^{-1}(F-V) x-\xi^{T} V^{-1} f(x, y) \\
& =\left(\mathcal{R}_{0}-1\right) \xi^{T} x-\xi^{T} V^{-1} f(x, y) .
\end{aligned}
$$

Since $\xi^{T} \geq 0, V^{-1} \geq 0$, and $f(x, y) \geq 0$ in $\Omega$, the last term is non-positive. If $\mathcal{R}_{0} \leq 1$, then $\mathcal{L}^{\prime} \leq 0$ in $\Omega$, and thus $\mathcal{L}$ is a Lyapunov function for the model (1)-(5).

Theorem 4 Let $F, V$ and $f(x, y)$ be defined, respectively as in (20), (21), and (26). Suppose that $f(x, y) \geq 0, F \geq 0, V^{-1} \geq 0$ and $V^{-1} F$ is irreducible. Assume that the disease-free system $y^{\prime}=g(0, y)$ has a unique equilibrium $y=y_{0}>0$ that is globally asymptotically stable in $\Omega$. Then, the following results hold for the model (1)-(5):

(i) If $\mathcal{R}_{0}<1$, then, $D_{0}$ is globally asymptotically stable in $\Omega$.

(ii) If $\mathcal{R}_{0}>1$, then, $D_{0}$ is unstable in $\Omega$.

Proof By Theorem (3), $\mathcal{L}^{\prime}=\xi^{T} V^{-1} x$ is a Lyapunov function for the model (1)-(5) provided that $\mathcal{R}_{0}<1$. Since $V^{-1} F$ is irreducible and non-negative, it follows by PerronFrobenius theory that $\xi>0$. Hence, by (28), $\mathcal{L}^{\prime}=0$ implies that $\xi^{T} x=0$, and thus $x=0$. Using the global stability assumption for the disease-free system and the fact that $f(0, y)=0$, the only invariant set in $\Omega$ where $x=0$ is the singleton $\left\{D_{0}\right\}$. Thus, $D_{0}$ is globally asymptotically stable in $\Omega[19,27]$. If $\mathcal{R}_{0}>1$, then by $(28), \mathcal{L}^{\prime}=\left(\mathcal{R}_{0}-1\right) \xi^{T} x>0$ provided $x>0$ and $y=y_{0}$. By continuity $\mathcal{L}^{\prime}>0$ in a neighborhood of $D_{0}$. Solutions in $\Omega$ sufficiently close to $D_{0}$ move away from $D_{0}$, implying that $D_{0}$ is unstable.

\section{Existence of the endemic equilibrium point $\left(D_{1}\right)$}

To find conditions for the existence of an equilibrium for which FMD is endemic in the population, i.e., at least one of $S^{*}, E^{*}, I^{*}, R^{*}, P^{*}$ is non-zero, denoted by $D_{1}=\left(S^{*}, E^{*}\right.$, $\left.I^{*}, R^{*}, P^{*}\right)$, Eqs. (1)-(5) are solved in terms of the force of infection at steady state $\left(T^{*}\right)$ given by

$$
T^{*}=\beta_{p} P^{*}+\beta_{I} I^{*}
$$


Setting the right-hand side of the Eqs. (1)-(5) to zero gives

$$
\begin{aligned}
S^{*} & =\frac{\Lambda(\sigma+\mu)(\mu+v+\gamma)(\mu+\omega)}{\left(T^{*}+\mu\right)(\sigma+\mu)(\mu+v+\gamma)(\mu+\omega)-\omega \gamma \sigma T^{*}}, \\
E^{*} & =\frac{\Lambda(\mu+v+\gamma)(\mu+\omega) T^{*}}{\left(T^{*}+\mu\right)(\sigma+\mu)(\mu+v+\gamma)(\mu+\omega)-\omega \gamma \sigma T^{*}}, \\
I^{*} & =\frac{\Lambda \sigma(\mu+\omega) T^{*}}{\left(T^{*}+\mu\right)(\sigma+\mu)(\mu+v+\gamma)(\mu+\omega)-\omega \gamma \sigma T^{*}}, \\
R^{*} & =\frac{\Lambda \sigma \gamma T^{*}}{\left(T^{*}+\mu\right)(\sigma+\mu)(\mu+v+\gamma)(\mu+\omega)-\omega \gamma \sigma T^{*}}, \\
P^{*} & =\frac{\lambda_{p} T^{*} \Lambda(\mu+\omega)(\sigma+\mu+v+\gamma)}{\left(T^{*}+\mu\right)(\sigma+\mu)(\mu+v+\gamma)(\mu+\omega) \delta_{p}-\omega \gamma \sigma \delta_{p} T^{*}} .
\end{aligned}
$$

Substituting (30) into (29) gives

$$
\begin{aligned}
T^{*}= & \frac{\beta_{p} \lambda_{p} T^{*} \Lambda(\mu+\omega)(\sigma+\mu+v+\gamma)}{\left(T^{*}+\mu\right)(\sigma+\mu)(\mu+v+\gamma)(\mu+\omega) \delta_{p}-\omega \gamma \sigma \delta_{p} T^{*}} \\
& +\frac{\beta_{I} \Lambda \sigma(\mu+\omega) T^{*}}{\left(T^{*}+\mu\right)(\sigma+\mu)(\mu+v+\gamma)(\mu+\omega)-\omega \gamma \sigma T^{*}},
\end{aligned}
$$

which results into a polynomial $b_{2}\left(T^{*}\right)^{2}+b_{1} T^{*}+b_{0}=0$, where

$$
\left\{\begin{array}{r}
b_{2}=\delta_{p}(\mu(\mu+v+\gamma)(\sigma+\mu+\omega)+\sigma \omega(v+\mu))\{\Lambda(\sigma+\mu+v+\gamma)(\mu+\omega) \\
+\mu(\mu+v+\gamma)(\sigma+\mu+\omega)+\sigma \omega(v+\mu)\} \\
b_{1}=2 \delta_{p} \mu \omega \gamma \sigma((\sigma+\mu)(\mu+v+\gamma)(\mu+\omega))^{2}(\mu(\mu+v+\gamma)(\sigma+\mu+\omega) \\
+\sigma \omega(v+\mu))\left\{\frac{2 \mathcal{R}_{0}\left(1-\frac{\mathcal{R}_{0}}{2}\right)+\mathcal{R}_{p}\left(1-\mathcal{R}_{0}\right)}{2 \mathcal{R}_{0}}\right\} \\
+\Lambda \delta_{p} \mu(\sigma+\mu)(\mu+v+\gamma)(\mu+\omega)^{2}(\sigma+\mu \\
+v+\gamma)\left\{\frac{\left(1-\mathcal{R}_{0}\right)\left(\mathcal{R}_{0}+\mathcal{R}_{p}\right)}{\mathcal{R}_{0}}\right\}, \\
\delta_{p}((\sigma+\mu)(\mu+v+\gamma)(\mu+\omega) \mu)^{2}\left\{\frac{\left(1-\mathcal{R}_{0}\right)\left(\mathcal{R}_{0}+\mathcal{R}_{p}\right)}{\mathcal{R}_{0}}\right\}
\end{array}\right.
$$

with $\mathcal{R}_{p}$ defined as in (23). It is worth noting that the coefficient $b_{2}$ is always positive and $b_{0}$ is positive (negative) if $\mathcal{R}_{0}$ is less than (greater than) unity, respectively. If $\mathcal{R}_{0}<1$, then $b_{1}>0$ and $b_{0}>0$, and no positive endemic equilibrium point exists. If $\mathcal{R}_{0}=1$, then $b_{1}>0$ and $b_{0}=0$. Note, the root $T^{*}=0$ when $\mathcal{R}_{0}=1$ corresponds to the disease-free equilibrium point $D_{0}$ whose stability has already been analysed. In this case, there is no positive endemic equilibrium point. If $\mathcal{R}_{0}>1$, regardless of the sign of $b_{1}$ there is one positive endemic equilibrium point which exists. This result is summarized below.

Lemma 2 The FMD model (1)-(5) has a unique positive endemic equilibrium point whenever $\mathcal{R}_{0}>1$ and no positive endemic equilibrium point otherwise.

\section{Local stability of the endemic equilibrium point $\left(D_{1}\right)$}

The Center Manifold Theorem is used to establish the local asymptotic stability of the endemic equilibrium point [12]. To apply this theorem, the following simplifications and change of variables are made first. Let $S=x_{1}, E=x_{2}, I=x_{3}, R=x_{4}$ and $P=x_{5}$. Further, by using vector notation $X=\left(x_{1}, x_{2}, x_{3}, x_{4}, x_{5}\right)^{T}$, the FMD model (1)-(5) can be written in the form $\frac{d X}{d t}=F(X)$, with $F=\left(f_{1}, f_{2}, f_{3}, f_{4}, f_{5}\right)^{T}$ as follows: 


$$
\begin{aligned}
& \frac{d x_{1}}{d t}=f_{1}=\Lambda+\omega x_{4}-\left(\beta_{p} x_{5}+\beta_{I} x_{3}\right) x_{1}-\mu x_{1}=0 \\
& \frac{d x_{2}}{d t}=f_{2}=\left(\beta_{p} x_{5}+\beta_{I} x_{3}\right) x_{1}-(\sigma+\mu) x_{2}=0, \\
& \frac{d x_{3}}{d t}=f_{3}=\sigma x_{2}-(\nu+\mu+\gamma) x_{3}=0, \\
& \frac{d x_{4}}{d t}=f_{4}=\gamma x_{3}-(\mu+\omega) x_{4}=0 \\
& \frac{d x_{5}}{d t}=f_{5}=\lambda_{p}\left(x_{3}+x_{2}\right)-\delta_{p} x_{6}=0 .
\end{aligned}
$$

After linearizing the system at disease-free equilibrium point, the Jacobian matrix at $D_{0}$ is given as

$$
\mathrm{J}_{\left(\mathrm{D}_{0}\right)}=\left(\begin{array}{ccccc}
-\mu & 0 & -\beta_{I} \frac{\Lambda}{\mu} & \omega & -\beta_{p} \frac{\Lambda}{\mu} \\
0 & -(\sigma+\mu) & \beta_{I} \frac{\Lambda}{\mu} & 0 & \beta_{p} \frac{\Lambda}{\mu} \\
0 & \sigma & -(\mu+\nu+\gamma) & 0 & 0 \\
0 & 0 & \gamma & -(\mu+\omega) & 0 \\
0 & \lambda_{p} & \lambda_{p} & 0 & -\delta_{p}
\end{array}\right) .
$$

Let $\phi^{*}$ be the bifurcation parameter. Consider $\phi^{*}=\beta_{p}^{*}$ as the bifurcation parameter. From (22), when $\mathcal{R}_{0}=1, \beta_{p}^{*}$ is determined as

$$
\beta_{p}^{*}=\frac{\delta_{p}\left[\mu(\sigma+\mu)(\mu+v+\gamma)-\beta_{I} \Lambda \sigma\right]}{\Lambda \lambda_{p}(\sigma+\mu+v+\gamma)},
$$

The Jacobian matrix for the transformed system with $\phi^{*}$ has a simple zero eigenvalue [12]. Therefore, the Center Manifold Theorem can be used to analyze the stability of the system near $\phi^{*}$ where by the local stability of $D_{1}$ of the transformed system is the same as the stability of $D_{0}$ of the original system for $\phi^{*}$ near $\phi^{*}$. That is, eigenvectors of $J\left(D_{1}\right) \mid \phi_{p}^{*}$ are equal to eigenvectors of $J\left(D_{0}\right) \mid \phi_{p}^{*}$. The Jacobian matrix for the transformed system has left and right eigenvectors associated with a simple zero eigenvalue. Let the left and right eigenvectors, respectively, be denoted by $m$ and $n$. The left eigenvector is given by $m=\left(m_{1}, m_{2}, m_{3}, m_{4}, m_{5}\right)$ where

$$
\left(\begin{array}{lllll}
m_{1} & m_{2} & m_{3} & m_{4} & m_{5}
\end{array}\right)\left(\begin{array}{ccccc}
-\mu & 0 & -\beta_{I} \frac{\Lambda}{\mu} & \omega & -\beta_{p} \frac{\Lambda}{\mu} \\
0 & J_{[2,2]} & \beta_{I} \frac{\Lambda}{\mu} & 0 & \beta_{p} \frac{\Lambda}{\mu} \\
0 & \sigma & -J_{[3,3]} & 0 & 0 \\
0 & 0 & \gamma & -(\mu+\omega) & 0 \\
0 & \lambda_{p} & \lambda_{p} & 0 & -\delta_{p}
\end{array}\right)=0 .
$$

Here, $J_{[2,2]}=\sigma+\mu$ and $J_{[3,3]}=\mu+v+\gamma$. Solving (36), we find $m_{1}=0, m_{2}=m_{2}>0$, $m_{3}=\Lambda \frac{\left(\beta_{I} \delta_{p}+\lambda_{p} \beta_{p}\right)}{\mu \delta_{p}(\mu+v+\gamma)} m_{2}>0, m_{4}=0$, and $m_{5}=\frac{\beta_{p} \Lambda m_{2}}{\mu \delta_{p}}>0$. The right eigenvector denoted by $n=\left(n_{1}, n_{2}, n_{3}, n_{4}, n_{5}\right)^{T}$ is given by

$$
\left(\begin{array}{ccccc}
-\mu & 0 & -\beta_{I} \frac{\Lambda}{\mu} & \omega & -\beta_{p} \frac{\Lambda}{\mu} \\
0 & -(\sigma+\mu) & \beta_{I} \frac{\Lambda}{\mu} & 0 & \beta_{p} \frac{\Lambda}{\mu} \\
0 & \sigma & -(\mu+v+\gamma) & 0 & 0 \\
0 & 0 & \gamma & -(\mu+\omega) & 0 \\
0 & \lambda_{p} & \lambda_{p} & 0 & -\delta_{p}
\end{array}\right)\left(\begin{array}{l}
n_{1} \\
n_{2} \\
n_{3} \\
n_{4} \\
n_{5}
\end{array}\right)=0 .
$$

This gives $n_{1}=\frac{-1}{\mu \sigma(\mu+\omega)}\{\mu(\mu+v+\gamma)(\mu+\omega+\sigma)+\sigma \omega(\mu+v)\} n_{3}<0, n_{2}=$ $\frac{(\mu+v+\gamma) n_{3}}{\sigma}>0, n_{3}=n_{3}>0, n_{4}=\frac{\gamma n_{3}}{\mu+\omega}>0$ and $n_{5}=\frac{\lambda_{p}(\mu+v+\gamma+\sigma) n_{3}}{\delta_{p} \sigma}>0$. The stability 
of the system around the equilibrium point is determined by the sign of the bifurcation coefficients $a$ and $b$ where

$$
a=\sum_{k, i, j=1}^{5} m_{k} n_{i} n_{j} \frac{\partial^{2} f_{k}(0,0)}{\partial x_{i} \partial x_{j}} \text { and } b=\sum_{k, i=1}^{5} m_{k} n_{i} \frac{\partial^{2} f_{k}(0,0)}{\partial x_{i} \partial \phi^{*}} .
$$

For $a$, since $m_{1}=0$ only values of $k=2$ will be considered.

When $k=2$, we have $\frac{\partial^{2} f_{2}(0,0)}{\partial x_{1} \partial x_{5}}=\frac{\partial^{2} f_{4}(0,0)}{\partial x_{5} \partial x_{1}}=\beta_{p}$, and $\frac{\partial^{2} f_{2}(0,0)}{\partial x_{1} \partial x_{3}}=\frac{\partial^{2} f_{2}(0,0)}{\partial x_{3} \partial x_{1}}=\beta_{I}$. Thus,

$$
\begin{aligned}
a= & 2 n_{1} m_{2}\left(\beta_{p} n_{5}+\beta_{I} n_{3}\right) \\
= & \frac{-2 m_{2} n_{3}^{2}\left(\lambda_{p} \beta_{p}(\mu+\nu+\gamma+\sigma)+\beta_{I} \delta_{p} \sigma\right)}{\mu \sigma^{2} \delta_{p}(\mu+\omega)}\{\mu(\mu+\nu+\gamma) \\
& (\mu+\omega+\sigma)+\sigma \omega(\mu+v)\}<0 .
\end{aligned}
$$

From (38), it can be seen that the only non-vanishing partial derivative at the disease-free equilibrium point is for $k=2$ when $i=5$, i.e., $\frac{\partial^{2} f_{2}(0,0)}{\partial x_{5} \partial \beta_{p}}=x_{1}=\frac{\Lambda}{\mu}$. Thus, $b=m_{2} n_{5} x_{1}=$ $\frac{\Lambda \lambda_{p}(\mu+\nu+\gamma+\sigma) m_{2} n_{3}}{\mu \delta_{p} \sigma}>0$. Since $a<0$ and $b>0$, by Center Manifold Theorem [12], we have established the following result:

Lemma 3 The unique endemic equilibrium point guaranteed by the Center Manifold Theorem is locally asymptotically stable when $\mathcal{R}>1$.

\section{Analysis of optimal control measures for the environmental transmission of}

\section{FMD}

Time-dependent preventive efforts $\left(u_{1}\right)$ and $\left(u_{2}\right)$ are introduced into the model (1)-(5) as controls to curtail the environmental transmission of FMD in cattle. The model (1)-(5) then becomes

$$
\begin{aligned}
& \frac{d S}{d t}=\Lambda+\omega R-\left(1-u_{1}\right)\left[\beta_{p} P+\beta_{I} I\right] S-\left(\mu+u_{1}\right) S, \\
& \frac{d E}{d t}=\left(1-u_{1}\right)\left[\beta_{p} P+\beta_{I} I\right] S-(\sigma+\mu) E, \\
& \frac{d I}{d t}=\sigma E-(\nu+\mu+\gamma) I, \\
& \frac{d R}{d t}=\gamma I+u_{1} S-(\mu+\omega) R, \\
& \frac{d P}{d t}=\lambda_{p}(I+E)-\left(\delta_{p}+u_{2}\right) P,
\end{aligned}
$$

where the control function $u_{1}(t) \in[0,1]$ is aimed at reducing the transmission rates $\beta_{p}$ and $\beta_{I}$. This is done by using the vaccination strategy. The control function $u_{2}(\mathrm{t}) \in[0,1]$ is a preventive measure which is aimed at increasing the decay rate $\delta_{p}$. This is through decontamination using disinfectants. The objective function for our minimization problem is given by

$$
J\left(u_{1}, u_{2}\right)=\int_{0}^{T}\left(B_{1} E(t)+B_{2} u_{1}^{2}+B_{3} u_{2}^{2}\right) d t,
$$

where $B_{1}, B_{2}$, and $B_{3}$ are positive weights attached to the advantages of controlling cattle infected with FMD over a finite period of time $T$. The main goal is to minimize the number of those infected while at the same time minimizing the cost of implementing the controls. In this respect, we seek optimal controls $u_{1}^{*}$ and $u_{2}^{*}$ such that 


$$
J\left(u_{1}^{*}, u_{2}^{*}\right)=\min \left\{\left(u_{1}, u_{2}\right) \mid u_{1}, u_{2} \in U\right\}
$$

subject to (40), where

$$
U=\left\{\left(u_{1}(t), u_{2}(t)\right) \in\left[L^{2}(0, T)\right]^{2} \mid 0 \leq u_{1} \leq 1,0 \leq u_{2} \leq 1, t \in[0, T]\right\} .
$$

The necessary conditions that an optimal control must satisfy come from the Pontryagin's Maximum Principle [23]. The basic framework of an optimal control problem is to prove the existence of the optimal control and then characterize the optimal control through the optimality system.

\section{Existence of an optimal control}

Let $u=\left(u_{1}, u_{2}\right) \in\left[L^{2}(0, T)\right]^{2}$. Then, a reduced cost function corresponding to (41) is defined as

$$
J\left(u, E^{u}\right)=\int_{0}^{T}\left(B_{1} E(t)+B_{2} u_{1}^{2}+B_{3} u_{2}^{2}\right) d t, \quad u \in U .
$$

\section{Lemma $4 U$ is closed and convex.}

Proof First, we prove that $U$ is closed. For this purpose, suppose that $u_{n} \longrightarrow u^{*}$ in $L^{2}(0, T)$ for $u_{n} \in U$, but $u^{*} \notin U$, i.e., $u^{*}<0$ or $u^{*}>1$ on a set of positive measure. Then by taking $u^{*}<0$, it follows from Lebesgue measure methods that there exists $\epsilon>0$ and a positive measure set $(0, r) \subset(0, T)$ so that $u^{*} \leq 0-\epsilon$ on $(0, r)$. This implies that

$$
\int_{0}^{T}\left(u_{n}-u^{*}\right)^{2} \geq \int_{0}^{r}\left(u_{n}-u^{*}\right)^{2} \geq \int_{0}^{r}\left(0-u^{*}\right)^{2} \geq \int_{0}^{r} \epsilon^{2}>0,
$$

a contradiction. Thus, $U$ is closed. Next, we prove convexity of $U$. First, we observe that since $\left(u_{1}, u_{2}\right) \in U$, we have

$$
0 \leq u_{1} \leq 1
$$

and

$$
0 \leq u_{2} \leq 1 .
$$

Multiplying (46) by $a$ and (47) by $(1-a)$ we get

$$
0 \leq a u_{1} \leq a,
$$

and

$$
0 \leq(1-a) u_{2} \leq(1-a) .
$$

Adding up Eqs. 48 and (49) gives

$$
0 \leq a u_{1}+(1-a) u_{2} \leq 1 .
$$

Thus, $a u_{1}+(1-a) u_{2} \in U$ and hence, $U$ is convex.

Theorem 5 There exists an optimal pair $\left(\left(u_{1}^{*}, u_{2}^{*}\right), E^{u^{*}}\right)$ to the optimization problem (42).

Proof Let

$$
d=\sup _{u \in U} J\left(u, E^{u}\right) .
$$


Then, for any $n \in \mathbb{N}$, there exists $u_{n} \in U$ such that

$$
d-\frac{1}{n}<J\left(u_{n}, E^{u_{n}}\right) \leq d .
$$

Since $U$ is a bounded subset of $L^{2}(0, T)$, it follows from Bolzano-Weierstrass theorem (every bounded sequence has a convergent subsequence), that there exists a subsequence $\left\{u_{n_{k}}\right\}_{k \in \mathbb{N}}$ such that

$$
u_{n_{k}} \longrightarrow u^{*}
$$

weakly in $L^{2}(0, T)$. Theorem 2 shows that the set of state variables is bounded. Therefore, there exists a subsequence $\left\{E^{u_{n_{k}}}\right\}_{k \in \mathbb{N}}$ such that

$$
E^{u_{n_{k}}} \longrightarrow E^{u^{*}} \text { in } C([0, T]) \text {. }
$$

From (52), we get

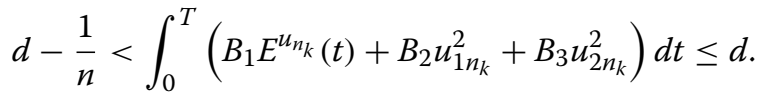

By (53) and (54), passing to the limit in (55), we obtain that

$$
d=\int_{0}^{T}\left(B_{1} E^{u^{*}}(t)+B_{2}\left(u_{1}^{*}\right)^{2}+B_{3}\left(u_{2}^{*}\right)^{2}\right) d t
$$

that is, $\left(\left(u_{1}^{*}, u_{2}^{*}\right), E^{u^{*}}\right)$ is an optimal pair where $u_{1}^{*}$ and $u_{2}^{*}$ are optimal controls for (42).

\section{Optimality system}

In this section, the optimality system using results from Lewis and Syrmos [20] is presented. The optimality system can be used to compute values for the optimal control pair. To do this, we begin by defining the Hamiltonian as follows

$$
\begin{aligned}
\mathcal{H}(X, U, \lambda)= & \left(B_{1} E(t)+B_{2} u_{1}^{2}+B_{3} u_{2}^{2}\right) \\
& +\lambda_{1}\left\{\Lambda+\omega R-\left(1-u_{1}\right)\left[\beta_{I} I+\beta_{p} P\right] S-\left(u_{1}+\mu\right) S\right\} \\
& +\lambda_{2}\left\{\left(1-u_{1}\right)\left[\beta_{I} I+\beta_{p} P\right] S-(\sigma+\mu) E\right\} \\
& +\lambda_{3}\{\sigma E-(v+\mu+\gamma) I\}+\lambda_{4}\left\{\gamma I+u_{1} S-(\mu+\omega) R\right\} \\
& +\lambda_{5}\left\{\lambda_{p}(I+E)-\left(\delta_{p}+u_{2}\right) P\right\}
\end{aligned}
$$

where $\lambda_{i}, i=1,2, \ldots ., 5$ are adjoint or co-state variables corresponding to the state variables $S, E, I, R$, and $P$, respectively.

Theorem 6 Let $\left(u_{1}^{*}, u_{2}^{*}\right)$ be an optimal control pair to (41) over $U$ with corresponding optimal states $S^{u^{*}}, E^{u^{*}}, I^{u^{*}}, R^{u^{*}}$, and $P^{u^{*}}$ of the system (40) that minimizes (41) over $U$. Then, there exist adjoint functions $\lambda_{i}, i=1,2, \ldots ., 5$ satisfying

$$
\begin{aligned}
\frac{\partial \lambda_{1}}{\partial t} & =\left(\lambda_{1}-\lambda_{2}\right)\left(1-u_{1}\right)\left[\beta_{I} I+\beta_{p} P\right]+\lambda_{1} \mu+u_{1}\left(\lambda_{1}-\lambda_{4}\right), \\
\frac{\partial \lambda_{2}}{\partial t} & =-B_{1}+\lambda_{2}(\sigma+\mu)-\lambda_{3} \sigma-\lambda_{5} \lambda_{p}, \\
\frac{\partial \lambda_{3}}{\partial t} & =\lambda_{3}(v+\mu+\gamma)-\lambda_{4} \gamma-\lambda_{5} \lambda_{p}, \frac{\partial \lambda_{4}}{\partial t}=-\lambda_{1} \omega+\lambda_{4}(\mu+\omega), \\
\frac{\partial \lambda_{5}}{\partial t} & =\left(\lambda_{1}-\lambda_{2}\right) \beta_{p}\left(1-u_{1}\right) S+\lambda_{5}\left(u_{2}+\delta_{p}\right),
\end{aligned}
$$


with transversality condition $\lambda_{i}(T)=0, i=1,2, \ldots ., 5$. Further, the following characterization holds:

$$
u_{1}^{*}=\max \left\{0, \min \left(1, \hat{u}_{1}(t)\right)\right\}, u_{2}^{*}=\max \left\{0, \min \left(1, \hat{u}_{2}(t)\right)\right\},
$$

where

$$
\hat{u}_{1}=\frac{\left(\beta_{I} I+\beta_{p} P\right)\left(\lambda_{2}-\lambda_{1}\right) S+\left(\lambda_{1}-\lambda_{4}\right) S}{2 B_{2}}, \hat{u}_{2}=\frac{\lambda_{5} P}{2 B_{3}} .
$$

Proof Pontryagin's maximum principle guarantees existence of $\lambda_{i}, i=1,2, \ldots ., 5$ satisfying

$$
\begin{aligned}
& \frac{\partial \lambda_{1}}{\partial t}=-\frac{\partial \mathcal{H}}{\partial S}, \quad \frac{\partial \lambda_{2}}{\partial t}=-\frac{\partial \mathcal{H}}{\partial E}, \quad \frac{\partial \lambda_{3}}{\partial t}=-\frac{\partial \mathcal{H}}{\partial I}, \quad \frac{\partial \lambda_{4}}{\partial t}=-\frac{\partial \mathcal{H}}{\partial R}, \\
& \frac{\partial \lambda_{5}}{\partial t}=-\frac{\partial \mathcal{H}}{\partial P}, \quad \lambda_{i}(T)=0, \quad i=1,2,3,4,5 .
\end{aligned}
$$

Utilizing (57) and applying (61), we obtain

$$
\begin{aligned}
& \frac{\partial \lambda_{1}}{\partial t}=\left(\lambda_{1}-\lambda_{2}\right)\left(1-u_{1}\right)\left[\beta_{I} I+\beta_{p} P\right]+\lambda_{1} \mu+u_{1}\left(\lambda_{1}-\lambda_{4}\right), \\
& \frac{\partial \lambda_{2}}{\partial t}=-B_{1}+\lambda_{2}(\sigma+\mu)-\lambda_{3} \sigma-\lambda_{5} \lambda_{p}, \\
& \frac{\partial \lambda_{3}}{\partial t}=\lambda_{3}(\nu+\mu+\gamma)-\lambda_{4} \gamma-\lambda_{5} \lambda_{p}, \frac{\partial \lambda_{4}}{\partial t}=-\lambda_{1} \omega+\lambda_{4}(\mu+\omega), \\
& \frac{\partial \lambda_{5}}{\partial t}=\left(\lambda_{1}-\lambda_{2}\right) \beta_{p}\left(1-u_{1}\right) S+\lambda_{5}\left(u_{2}+\delta_{p}\right),
\end{aligned}
$$

with transversality condition $\lambda_{i}(T)=0, i=1,2, \ldots ., 5$. Differentiating (57) with respect to each of the admissible controls, we obtain

$$
\begin{aligned}
\frac{\partial \mathcal{H}}{\partial u_{1}} & =2 B_{2} u_{1}+\left(\beta_{I} I+\beta_{p} P\right)\left(\lambda_{1}-\lambda_{2}\right) S+\left(\lambda_{4}-\lambda_{1}\right) S, \\
\frac{\partial \mathcal{H}}{\partial u_{2}} & =2 B_{3} u_{2}-\lambda_{5} P .
\end{aligned}
$$

Setting the right-hand side of (63) to zero, we get the possible values $\hat{u}_{1}$ and $\hat{u}_{2}$ of the optimal controls $u_{1}^{*}$ and $u_{2}^{*}$ solving the following system.

$$
2 B_{2} \hat{u}_{1}+\left(\beta_{I} I+\beta_{p} P\right)\left(\lambda_{1}-\lambda_{2}\right) S+\left(\lambda_{4}-\lambda_{1}\right) S=0,2 B_{3} \hat{u}_{2}-\lambda_{5} P=0 .
$$

Solving (64), we find

$$
\hat{u}_{1}=\frac{\left(\beta_{I} I+\beta_{p} P\right)\left(\lambda_{2}-\lambda_{1}\right) S+\left(\lambda_{1}-\lambda_{4}\right) S}{2 B_{2}}, \hat{u}_{2}=\frac{\lambda_{5} P}{2 B_{3}} .
$$

The standard arguments on the controls are such that

$$
u_{1}^{*}=\left\{\begin{array}{l}
0 \quad \text { if } \hat{u}_{1} \leq 0, \\
\hat{u}_{1} \text { if } 0<\hat{u}_{1}<1, \\
1 \text { if } \hat{u}_{1} \geq 1,
\end{array}\right.
$$

where the value $u_{1}^{*}=1$ is a characteristic of a perfectly effective vaccine. However, this upper bound $\left(u_{1}^{*}=1\right)$ of this control may not be necessarily attainable. In a similar manner, we have

$$
u_{2}^{*}= \begin{cases}0 & \text { if } \hat{u}_{2} \leq 0, \\ \hat{u}_{2} & \text { if } 0<\hat{u}_{2}<1, \\ 1 & \text { if } \hat{u}_{2} \geq 1,\end{cases}
$$


where the upper bound of $u_{2}^{*}$ indicates that the contaminated environment is least likely to be responsible for the transmission of FMD, especially if disinfectants are used. From (66) and (67), we get

$$
u_{1}^{*}=\max \left\{0, \min \left(1, \hat{u}_{1}(t)\right)\right\}, u_{2}^{*}=\max \left\{0, \min \left(1, \hat{u}_{2}(t)\right)\right\} .
$$

The optimality system is given by the state system (40) with the initial conditions, the adjoint system (62) with the transversality conditions and the optimality condition (68). The optimal control is obtained by solving the optimality system using a gradient type algorithm with projection. By subdividing the interval $[0, T]$ into $n$ sub-intervals such that $n=\frac{T}{h}$, where $h$ is the step size, the solutions of the state system (40) and the adjoint system (62), respectively, are obtained by using Runge-Kutta fourth order schemes forward and backward in time. Denoting the solutions of (40), (62), and (68), respectively, by $x^{k}=$ $\left.\left\{S\left(t_{k}\right), E\left(t_{k}\right), I\left(t_{k}\right), R\left(t_{k}\right), P\left(t_{k}\right)\right\}, \lambda^{n-k}=\left\{\lambda_{1}\left(t_{n-k}\right), \lambda_{2}\left(t_{n-k}\right), \lambda_{3}\left(t_{n-k}\right), \lambda_{4}\left(t_{n-k}\right), \lambda_{5}\left(t_{n-k}\right)\right)\right\}$ and $u^{k}=\left\{u_{1}^{*}\left(t_{k}\right), u_{2}^{*}\left(t_{k}\right)\right\}$, where $t_{k}=k h$ and $k=0,1,2, \ldots ., n$. The algorithm for obtaining the optimal control is given as follows:

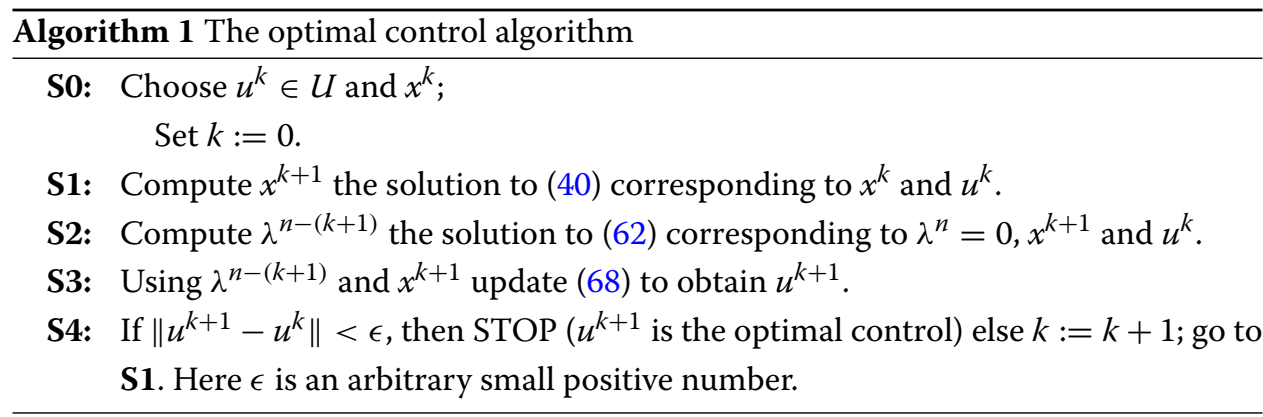

\section{Numerical simulations for optimal control}

Parameter values and initial model variables used are $\beta_{p}=0.007, \lambda_{p}=0.01, S(0)=1000$ cattle, $E(0)=1, I(0)=0, R(0)=0, P(0)=0$, and $\mathcal{R}_{0}=4.7837$. Other parameter values are in Table 1. The weight factors used are $B_{1}=$ UShs $10750, B_{2}=$ UShs 1000, and $B_{3}=$ UShs 1350. The values used here are intended only for theoretical purposes to investigate the effect of various control strategies. Three control strategies $A, B$, and $C$ are studied.

\section{Strategy A: optimal use of vaccination}

Here, only the control of vaccination $u_{1}$ is used to optimize the objective function (41) while the control of the environmental decontamination $u_{2}$ is set to zero. From Fig. 2a it can be observed that the control strategy led to a decrease in $E$ and $P$ as against an increase in the uncontrolled case. From Fig. 2b, it can be observed further that vaccination alone cannot reduce the number of pathogens in the environment to zero. This can lead to a tail of infection in the long run. The control profile is shown in Fig. 2c where it is observed that the optimal control of vaccination $u_{1}$ is at the upper bound till the time $T=27$ days before dropping to the lower bound. The result here show that if $100 \%$ of cattle in a herd of 1000 cattle are vaccinated within a period of 27 days of the outbreak, FMD transmission 
(a)

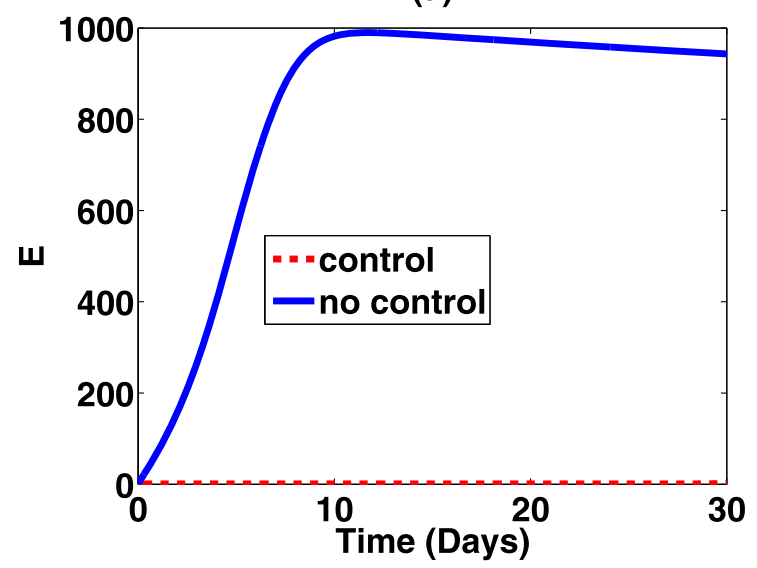

(b)

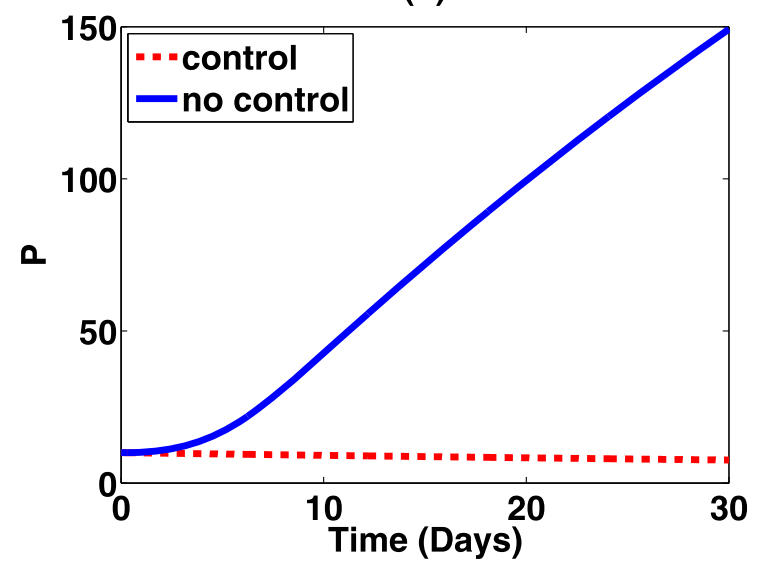

(c)

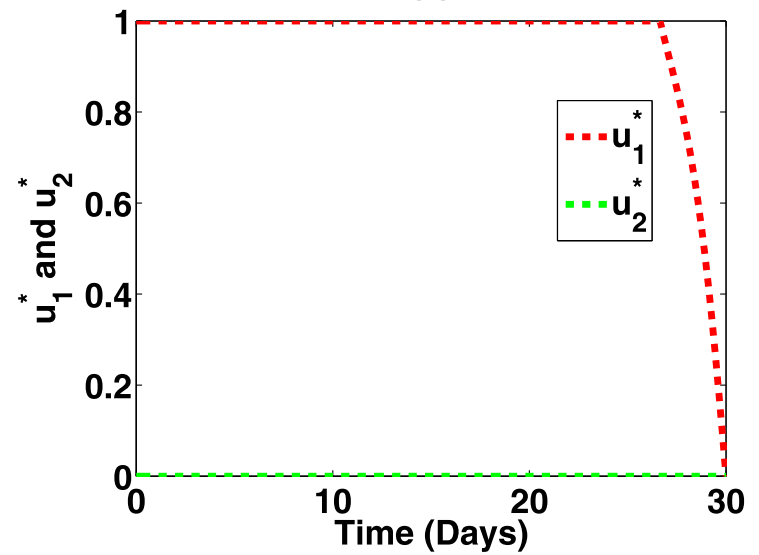

Fig. 2 a-c Optimal states and control profile for control strategy A

can be greatly reduced. The control of the environmental decontamination $u_{2}$ is at zero because in this strategy, only the control of vaccination $u_{1}$ is active.

\section{Strategy B: optimal use of environmental decontamination}

With this strategy, only the control of environmental decontamination $u_{2}$ is used to optimize the objective function (41) while the control of vaccination $u_{1}$ is set to zero. From 
Fig. 3a, the result show no big difference between $E$ with and without optimal control. In Fig. 3b, a big difference is observed between $P$ with and without optimal control. This strategy does not meet our objective which is minimizing the number of infections. The control profile is shown in Fig. 3c where the optimal control of environmental decontamination $u_{2}$ starts and stays at the upper bound for $T=12$ days, before dropping to the lower bound. The results here indicate that if an effort of $100 \%$ is put in decontaminating the environment from the 1st day to the 12th day, the transmission of FMD through a contaminated environment can be reduced. This strategy is less expensive as compared to strategy $A$ (optimal use of vaccination) as it takes 15 fewer days of the intervention. Figure 3c shows that control of vaccination $u_{1}$ is at zero for the entire period of intervention and this is due to the fact that in this strategy only the control of environmental decontamination $u_{2}$ is active.

\section{Strategy C: optimal use of both vaccination and environmental decontamination}

With this strategy, both vaccination $\left(u_{1}\right)$ and environmental decontamination $u_{2}$ are used to optimize the objective function (41). From Fig. $4 \mathrm{a}$ and b, the result show a significant difference in $E$ and $P$ with the optimal control strategy as compared to the case without control. The controlled trajectories show convergence to the disease-free equilibrium point. In Fig. 4c, the control profile, the control of vaccination $u_{1}$ is at the upper bound of $100 \%$ for 11 days and drops gradually until reaching the lower bound, while the control of environmental decontamination $u_{2}$ starts at the upper bound where it is maintained for 8 days before dropping slowly to the lower bound of $0 \%$ at the end of 30 days. This figure shows clearly how emergency vaccination and environmental decontamination can be used optimally to control the transmission of FMD through a contaminated environment.

\section{Discussion}

In this paper, a deterministic mathematical model describing the transmission dynamics of FMD in a contaminated environment was formulated and analysed. The model is shown to have a locally and globally asymptotically stable disease-free equilibrium point whenever its associated reproduction number is less than unity and has a unique, locally asymptotically stable endemic equilibrium point when $\mathcal{R}_{0}$ exceeds unity. The existence of a stable disease-free equilibrium point implies that the classical requirement of having the basic reproduction number less than unity is necessary and sufficient for the control of the transmission of FMD in a contaminated environment. The existence of a stable endemic equilibrium point when $\mathcal{R}_{0}$ exceeds unity indicates that the disease can persist in the livestock community if control measures are not put in place.

From the analysis of $\mathcal{R}_{0}$, it is shown that the transmission $\left(\beta_{p}\right.$ and $\left.\beta_{I}\right)$ and the shed$\operatorname{ding}\left(\lambda_{p}\right)$ rates have a potential of aggravating the disease if increased. Therefore, control measures such as vaccination, isolation, and quarantine are encouraged to minimize the aforementioned parameters. Similarly, the decay $\left(\delta_{p}\right)$ rate parameter is shown to reduce the incidence of the disease if increased. This implies that control measures (such as decontamination) that accelerate the decay rate of FMD in the environment can yield positive results in the control of the disease. Further, it is shown that the host-to-host route leads to more infections than the environment-to-host route. Thus, all farming practices that result into physical contacts of hosts like communal grazing and sharing of water points should be avoided. 
(a)

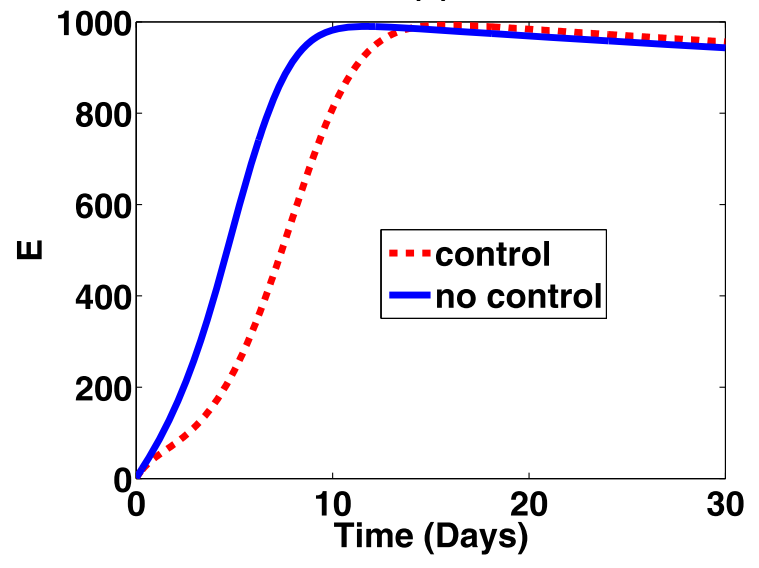

(b)

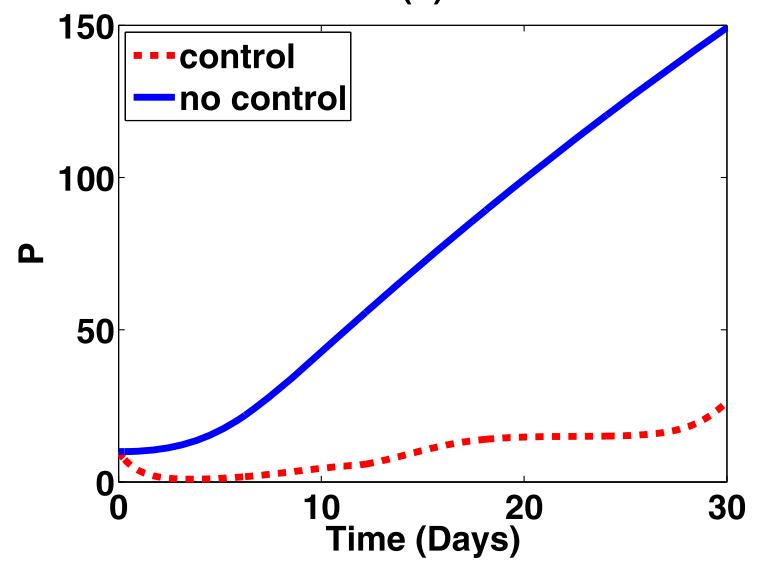

(c)

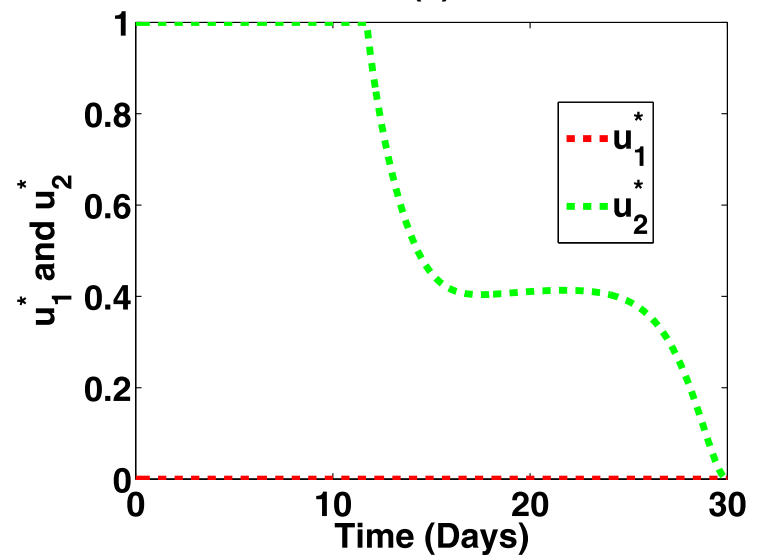

Fig. 3 a-c Optimal states and control profile for control strategy $B$

In the "Analysis of optimal control measures for the environmental transmission of FMD" section, the model was then transformed into an optimal control problem with an aim of minimizing the number of infected cattle at a minimal cost. Using the Pontryagin's Maximum Principle the optimality system was determined. Utilizing a gradient type 
(a)

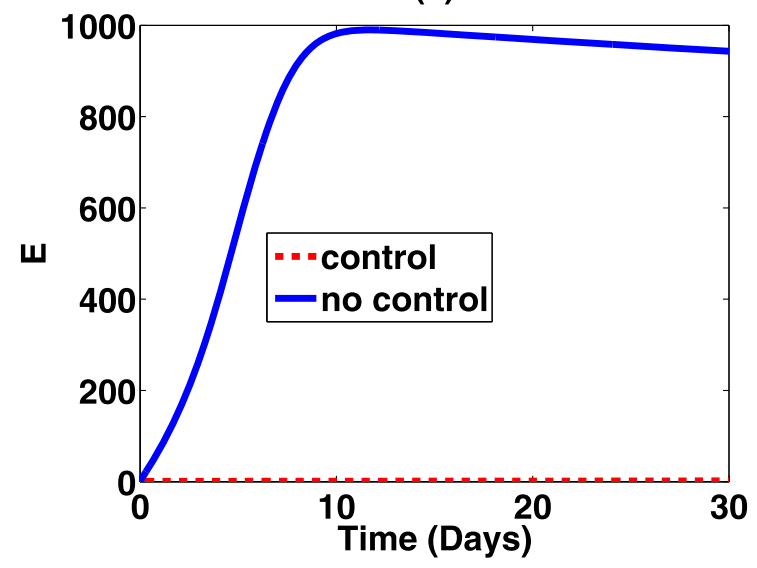

(b)

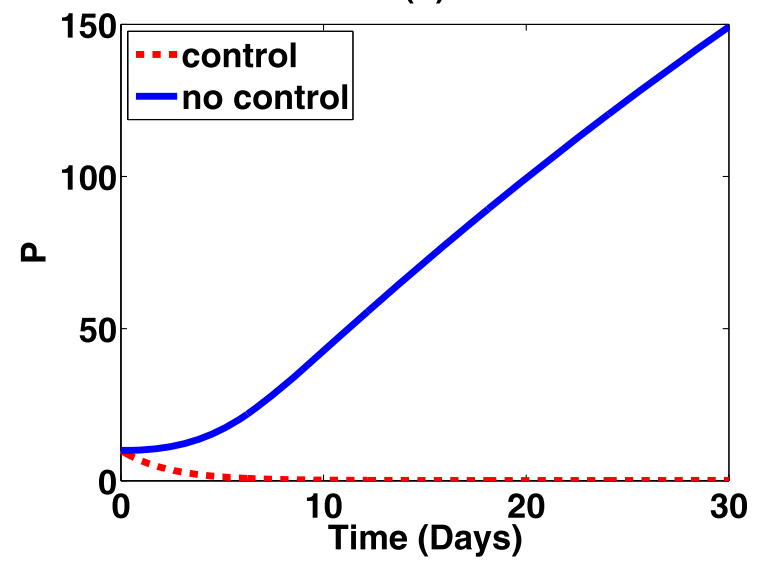

(c)

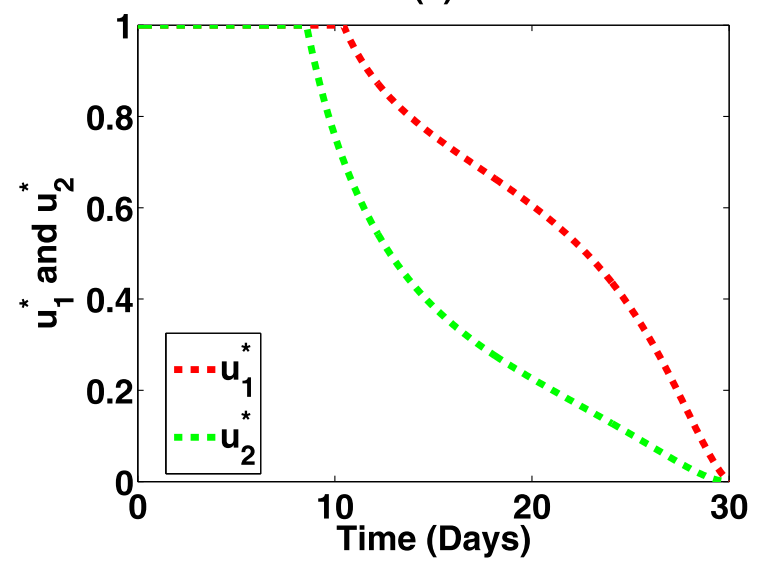

Fig. 4 a-c Optimal states and control profile for control strategy $C$

algorithm with projection, the optimality system was solved for three control strategies: optimal use of vaccination (Strategy $A$ ), environmental decontamination (Strategy $B$ ), and a combination of vaccination and environmental decontamination (Strategy $C$ ). Results of strategy $A$ (Fig. 2) show that vaccination alone can minimize the infections to zero. 
However, it does not reduce the number of pathogens in the environment to zero. This can lead to an environmental transmission in the long run especially when the vaccine is not perfect or if the vaccination coverage is not to the maximum.

From Fig. 3, it was noted that environmental decontamination alone has little impact on the disease prevalence. This is in line with a study by Bani-Yaghoub et al. [5], where it was discovered that environmental decontamination can reduce the value of $\mathcal{R}_{0}$ to less than unity, only when the environmental factors are the main cause of the infection. In this study, it has been shown that the host-to-host route is more dominant than the environment-to-host route rendering the single use of environmental decontamination a less effective strategy. Results of strategy $C$ suggest that when vaccination and environmental decontamination are used optimally, it is possible to attain the disease-free equilibrium point. This again is line with a study by Bani-Yaghoub et al. [5] where it was found out that elimination of endemic equilibrium through environmental decontamination is possible when it is applied in combination with other control measures. Strategy $C$ followed by $A$ are the best strategies in the control of FMD in the presence of environmental transmission.

From the results of this study, it can be concluded that it is possible to have both disease-free and endemic equilibria points for the model (1)-(5). In an FMD endemic area, regardless of the amount of FMDV in the environment and the number of infected cattle, the transmission of FMD can always be contained provided that the transmission, decay and shedding rates of the virus are simultaneously taken into consideration. In communities where resources are limited, a control strategy that involves optimal use of vaccination should be adopted with the best option being when vaccination is combined with environmental decontamination. The farmer can save more funds if the optimal control conditions discussed in this study are adhered to.

\section{Acknowledgments}

The authors are indebted to Prof. K.J. Huenink (Mama) and Prof. Pam Berns for proofreading the manuscript. The authors are grateful to the anonymous referee and reviewers for their valuable comments which led to the improvement of this manuscript.

\section{Authors' contributions}

FM drafted and analysed the manuscript. JYT, BNK, and HK supervised the study. MT participated in the analysis. All authors read and approved the final manuscript.

\section{Funding}

Francis Mugabi would like to thank The World Academy of Sciences (TWAS) for the financial support as tuition fees of his Master's program (grant no. 16-475RG/MATHS/AF/ACG). However, TWAS did not participate in the design of this manuscript.

\section{Availability of data and materials}

All data generated in this manuscript are included in the list of references.

\section{Competing interests}

The authors declare that they have no competing interests.

\section{Author details}

${ }^{1}$ College of Natural Sciences, Makerere University, Wandegeya, P. O. Box 7062 Kampala, Uganda. ${ }^{2}$ College of Veterinary Medicine, Animal Resources and Biosecurity, Makerere University, Wandegeya, P. O. Box 7062 Kampala, Uganda.

Received: 11 September 2019 Accepted: 22 November 2019

Published online: 19 December 2019

References

1. Alexandersen, S., Zhang, Z., Donaldson, A. I., Garland, A. J. M.: The pathogenesis and diagnosis of foot-and-mouth disease. J. Comp. Pathol. 129(1), 1-36 (2003) 
2. Andrus, D., Freeman, A., Eastwood, B.: Age distribution and herd life expectancy in lowa dairy herds. J. Dairy Sci. 53(6), 764-71 (1970)

3. Arzt, J, Juleff, N, Zhang, Z, Rodriguez, L. L: The pathogenesis of foot-and-mouth disease: Viral pathways in cattle. Transboundary Emerg. Dis. 58(4), 291-304 (2011)

4. Bachrach, H. L, Breese Jr, S. S, Callis, J. J, Hess, W. R, Patty, R. E: Inactivation of foot-and-mouth disease virus by pH and temperature changes and by formaldehyde. Proc. Soc. Exp. Biol. Med. 95(1), 147-152 (1957)

5. Bani-Yaghoub, M, Gautam, R, Döpfer, D, Kaspar, C, Ivanek, R: Effectiveness of environmental decontamination as an infection control measure. Epidemiol Infect. 140(3), 542-553 (2012)

6. Bani-Yaghoub, M, Gautam, R, Shuai, Z, van den Driessche, P, Ivanek, R: Reproduction numbers for infections with free-living pathogens growing in the environment. J. Biol. Dyn. 6(2), 923-940 (2012)

7. Barteling, S. J.: Development and performance of inactivated vaccines against foot and mouth disease. Rev. Sci. Tech.-Off. Int. Épizooties. 21(3), 577-583 (2002)

8. Belsham, G. J.: Distinctive features of foot-and-mouth disease virus, a member of the picornavirus family; aspects of virus protein synthesis, protein processing and structure. Prog. Biophys. Mol. Biol. 60(3), 241-260 (1993)

9. Bouma, A., de Jong, M. C. M., Kimman, T.: Transmission of pseudorabies virus within pig populations is independent of the size of the population. Prev. Vet. Med. 23(3-4), 163-172 (1995)

10. Breban, R., Drake, J. M., Stallknecht, D. E., Rohani, P.: The role of environmental transmission in recurrent avian influenza epidemics. PLoS. Comput. Biol. 5(4), e1000346 (2009)

11. Callis, J. J., et al.: Illustrated manual for the recognition and diagnosis of certain animal diseases. Technical report (1982)

12. Castillo-Chavez, C., Song, B.: Dynamical models of tuberculosis and their applications. Math. Biosci. Eng. 1(2), 361-404 (2004)

13. Doel, T. R.: Natural and vaccine-induced immunity to foot and mouth disease: the prospects for improved vaccines. Rev. Sci. Tech. (Int. Off. Epizootics). 15(3), 883 (1996)

14. Ferguson, N. M., Donnelly, C. A., Anderson, R. M.: The foot-and-mouth epidemic in Great Britain: pattern of spread and impact of interventions. Science. 292(5519), 1155-1160 (2001)

15. Golde, W. T., Pacheco, J. M., Duque, H., Doel, T., Penfold, B., Ferman, G. S., Gregg, D. R., Rodriguez, L. L.: Vaccination against foot-and-mouth disease virus confers complete clinical protection in 7 days and partial protection in 4 days: use in emergency outbreak response. Vaccine. 23(50), 5775-5782 (2005)

16. Hethcote, H. W.: The mathematics of infectious diseases. SIAM Rev. 42(4), 599-653 (2000)

17. Keeling, M. J., Woolhouse, M. E. J., Shaw, D. J., Matthews, L., Chase-Topping, M., Haydon, D. T., Cornell, S. J., Kappey, J., Wilesmith, J., Grenfell, B. T.: Dynamics of the 2001 UK foot and mouth epidemic: stochastic dispersal in a heterogeneous landscape. Science. 294(5543), 813-817 (2001)

18. Krug, P. W., Larson, C. R., Eslami, A. C., Rodriguez, L. L.: Disinfection of foot-and-mouth disease and african swine fever viruses with citric acid and sodium hypochlorite on birch wood carriers. Vet. Microbiol. 156(1-2), 96-101 (2012)

19. LaSalle, J. P.: Stability theory and invariance principles. In: Dynamical Systems, Vol. 1, pp. 211-222. Elsevier, (1976) https://doi.org/10.1016/B978-0-12-164901-2.50021-0

20. Lewis, F. L., Syrmos, V. L.: Optimal control. Wiley, New York (1995)

21. Morris, R. S., Wilesmith, J. W., Stern, M. W., Sanson, R. L., Stevenson, M. A.: Predictive spatial modelling of alternative control strategies for the foot-and-mouth disease epidemic in Great Britain. In: II International Symposium on Application of Modelling as an Innovative Technology in the Agri-Food Chain; MODEL-IT 566, pp. 337-347, (2001). https://doi.org/10.17660/actahortic.2001.566.43

22. Parham, P. E., Singh, B. K., Ferguson, N. M.: Analytic approximation of spatial epidemic models of foot and mouth disease. Theor. Popul. Biol. 73(3), 349-368 (2008)

23. Pontryagin, L. S.: Mathematical theory of optimal processes. CRC Press (1987)

24. Rohani, P., Breban, R., Stallknecht, D. E., Drake, J. M.: Environmental transmission of low pathogenicity avian influenza viruses and its implications for pathogen invasion. Proc. Natl. Acad. Sci. 106(25), 5-10369 (1036)

25. Shuai, Z., van den Driessche, P.: Global stability of infectious disease models using lyapunov functions. SIAM J. Appl. Math. 73(4), 1513-1532 (2013)

26. Tunc, C.: A study of the stability and boundedness of the solutions of nonlinear differential equations of the fifth order. Indian J. Pure Appl. Math. 33(4), 519-530 (2002)

27. Tunç, $\mathrm{C}:$ New results on the stability and boundedness of nonlinear differential equations of fifth order with multiple deviating arguments. Bull. Malays. Math. Sci. Soc. 36(3), 671-682 (2013)

28. van Bunnik, B. A. D., Katsma, W. E. A., Wagenaar, J. A., Jacobs-Reitsma, W. F., de Jong, M. C. M.: Acidification of drinking water inhibits indirect transmission, but not direct transmission of campylobacter between broilers. Prev. Vet. Med. 105(4), 315-319 (2012)

29. van den Driessche, P., Watmough, J: Reproduction numbers and sub-threshold endemic equilibria for compartmental models of disease transmission. Math. Biosci. 180(1), 29-48 (2002)

30. van Roermund, H. J. W., Eblé P.L., de Jong, M. C. M., Dekker, A.: No between-pen transmission of foot-and-mouth disease virus in vaccinated pigs. Vaccine. 28(28), 4452-4461 (2010)

31. Velkers, F. C., Bouma, A., Stegeman, J. A., de Jong, M. C. M.: Oocyst output and transmission rates during successive infections with eimeria acervulina in experimental broiler flocks. Vet. Parasitol. 187(1), 63-71 (2012)

\section{Publisher's Note}

Springer Nature remains neutral with regard to jurisdictional claims in published maps and institutional affiliations. 